\title{
Study of the $\pi \pi$ system in a finite volume
}

\author{
Dan Zhou and Hua-Xing Chen* \\ School of Physics and Nuclear Energy Engineering and International Research Center \\ for Nuclei and Particles in the Cosmos, Beihang University, Beijing 100191, China \\ *hxchen@buaa.edu.cn \\ E. Oset \\ Departamento de Física Teórica and IFIC, Centro Mixto Universidad de Valencia-CSIC \\ Institutos de Investigación de Paterna, Aptdo. 22085, 46071 Valencia, Spain \\ oset@ific.uv.es
}

Published 16 April 2014

\begin{abstract}
We evaluate energy levels of the $\pi \pi$ system in the $\rho$ channel in a finite volume using chiral unitary theory. We investigate $\pi \pi$ phase shifts and $\rho$ meson properties using Lattice QCD data with high precision. We also shortly discuss the $\pi K$ system in the $K^{*}$ channel in a finite volume.
\end{abstract}

Keywords: Multichannel scattering; lattice QCD calculation; meson-meson interaction.

PACS Numbers: 11.80.Gw, 12.38.Gc, 12.39.Fe, 13.75.Lb

\section{Introduction}

One of the challenging tasks of lattice QCD is to determine hadron spectra. Many efforts have been devoted to it. ${ }^{1-12}$ Particularly, Lüscher's approach is playing gradually a more important role. ${ }^{13,14}$ It has been applied to study the $\rho$ meson and the $K^{*}$ meson. ${ }^{13-23}$

In this paper ${ }^{24}$ we shall investigate the $\rho$ meson using the method proposed in Ref. 25. In this reference Lüscher's approach is simplified and improved by keeping the full relativistic two-body propagator, and is extended to coupled channels. This method has been used to study the $\kappa$ resonance in the $K \pi$ channel, the $D K$ and $\eta D_{s}$ system, the meson-baryon interaction and the $\pi \rho$ scattering, etc. ${ }^{26-35}$

To use this method, first we need to use the chiral unitary model to investigate the $\pi \pi$ scattering in $P$-waves. This has been done in Refs. 36, 37. Based on these results, we shall evaluate energy levels of the $\pi \pi$ system in the $\rho$ channel in a finite

This is an Open Access article published by World Scientific Publishing Company. It is distributed under the terms of the Creative Commons Attribution 3.0 (CC-BY) License. Further distribution of this work is permitted, provided the original work is properly cited. 
volume. By doing this we can establish a connection with lattice results which run for heavier pion masses than physical ones. ${ }^{21}$ We shall also discuss the $\pi K$ scattering in $P$-waves in a finite volume. The chiral unitary approach has been applied to investigate it in Refs. 36, 38, and the relevant lattice results are shown in Refs. 22, 23.

The paper proceeds as follows: in Sec. 2 we introduce the chiral unitary approach for the $\pi \pi$ scattering in $P$-waves together with its coupled $K \bar{K}$ channel, both in an infinite space and in a finite volume; in Sec. 3 we evaluate their energy levels and apply Lüscher formalism to get the relevant phase shifts; in Sec. 4 we discuss the $\pi K$ system in a finite volume; Sec. 5 is a short summary.

\section{Formalism for the $\pi \pi$ System}

The $P$-wave $\pi \pi$ scattering amplitude has been obtained in Refs. 37, 39, 40 using the chiral unitary model. They use the coupled channel Bethe-Salpeter equations:

$$
\mathbf{T}=(1-\mathbf{V G})^{-1} \mathbf{V}
$$

The relevant $V$ matrices for $\pi \pi$ and $K \bar{K}$ scattering have been studied in Ref. 37:

$$
\begin{aligned}
& V_{\pi \pi, \pi \pi}^{I=1}=T_{\pi^{+} \pi^{-}, \pi^{+} \pi^{-}}=-\frac{2 p_{\pi}^{2}}{3 f^{2}}\left(1+\frac{2 G_{V}^{2}}{f^{2}} \frac{s}{M_{\rho}^{2}-s}\right), \\
& V_{\pi \pi, K \bar{K}}^{I=1}=\sqrt{2} T_{\pi^{+} \pi^{-}, K^{+} K^{-}}=-\frac{\sqrt{2} p_{\pi} p_{K}}{3 f^{2}}\left(1+\frac{2 G_{V}^{2}}{f^{2}} \frac{s}{M_{\rho}^{2}-s}\right), \\
& V_{K \bar{K}, K \bar{K}}^{I=1}=T_{K^{+} K^{-}, K^{+} K^{-}}-T_{K^{+} K^{-}, K^{0} \bar{K}^{0}}=-\frac{p_{K}^{2}}{3 f^{2}}\left(1+\frac{2 G_{V}^{2}}{f^{2}} \frac{s}{M_{\rho}^{2}-s}\right) .
\end{aligned}
$$

where the states are defined in the isospin base:

$$
\begin{aligned}
|\pi \pi\rangle_{I=1} & =\frac{1}{2}\left|\pi^{+} \pi^{-}-\pi^{-} \pi^{+}\right\rangle \\
|K \bar{K}\rangle_{I=1} & =\frac{1}{\sqrt{2}}\left|K^{+} K^{-}-K^{0} \bar{K}^{0}\right\rangle
\end{aligned}
$$

In the chiral unitary model the $G$ function for the two-meson propagator is defined in the infinite space

$$
G\left(p^{2}\right)=i \int \frac{d^{4} q}{(2 \pi)^{4}} \frac{1}{q^{2}-m^{2}+i \epsilon} \frac{1}{(p-q)^{2}-M^{2}+i \epsilon},
$$

and calculated using the dimensional regularization:

$$
G_{i}(s)=\frac{1}{16 \pi^{2}}\left(-2+d_{i}+\sigma_{i}(s) \log \frac{\sigma_{i}(s)+1}{\sigma_{i}(s)-1}\right) .
$$


In this paper ${ }^{24}$ we shall study the $\rho$ meson in a finite volume. To do this we just use the following $G$ function which is defined in the finite box of side $L$ :

$$
\tilde{G}(s)-G(s)=\lim _{q_{\max } \rightarrow \infty}\left(\frac{1}{L^{3}} \sum_{q_{i}}^{q_{\max }} I\left(q_{i}\right)-\int_{q<q_{\max }} \frac{d^{3} q}{(2 \pi)^{3}} I(q)\right),
$$

where

$$
I\left(q_{i}\right)=\frac{1}{2 \omega_{1}(\vec{q}) \omega_{2}(\vec{q})} \frac{\omega_{1}(\vec{q})+\omega_{2}(\vec{q})}{E^{2}-\left(\omega_{1}(\vec{q})+\omega_{2}(\vec{q})\right)^{2}},
$$

and $\omega_{1,2}(\vec{q})=\sqrt{m_{1,2}^{2}+\vec{q}^{2}}$. The sum is given by $\vec{q}=\frac{2 \pi}{L} \vec{n}\left(\vec{n} \in \mathcal{Z}^{3}\right)$.

\section{Energy Levels for the $\pi \pi$ System}

Energy levels for the $\pi \pi$ System in a finite volume are obtained when the determinant of $1-$ VG is zero:

$$
\operatorname{Det}(1-\mathbf{V G})=1-V_{11} \tilde{G}_{1}(s)-V_{22} \tilde{G}_{2}(s)+\left(V_{11} V_{22}-V_{12}^{2}\right) \tilde{G}_{1}(s) \tilde{G}_{2}(s)=0 .
$$

We note that they are just the poles of the $\mathbf{T}$ matrix. Through this equation, we can quickly find that energy levels for $\pi \pi P$-wave scattering are functions of the cubic box size $L$.

The obtained energy levels are shown in Fig. 1 as functions of the box size $L$, where the left panel and the right panel are for the coupled channels and only for the single $\pi \pi$ channel, respectively. In these two figures the first and the second energy levels are almost the same, but the higher levels are different. This means the $K \bar{K}$ channel contributes here but it does not contribute much in the low-energy region.

Phase shifts can be obtained using these energy levels. Particularly, the $\pi \pi P$ wave phase shifts and the $T_{11}$ matrix are related to each other:

$$
T_{11}(E)=\frac{-8 \pi E}{p \cot \delta(p)-i p}
$$

where $p$ is the pion center-of-mass momentum. We show it in Fig. 2. The solid curve is taken from Ref. 37 using the chiral unitary approach in an infinite space. The
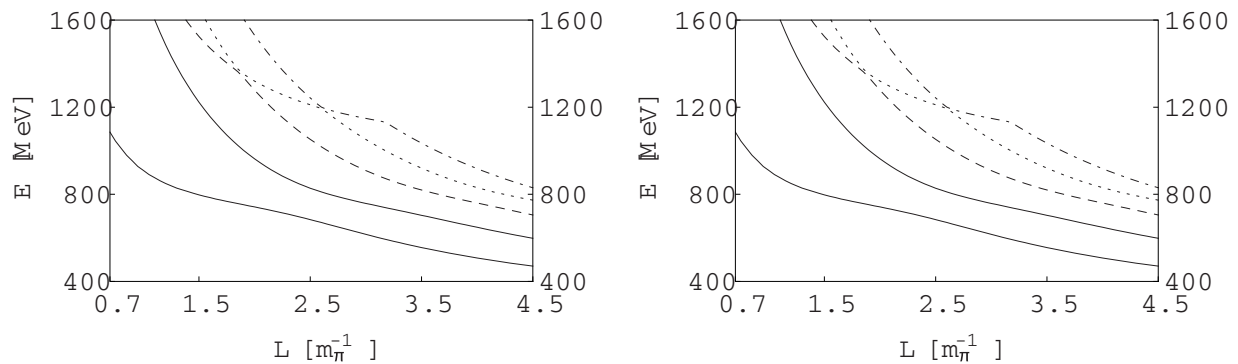

Fig. 1. Energy levels as functions of the box size $L$. The left panel and the right panel are for the coupled channels and only for the single $\pi \pi$ channel, respectively. 


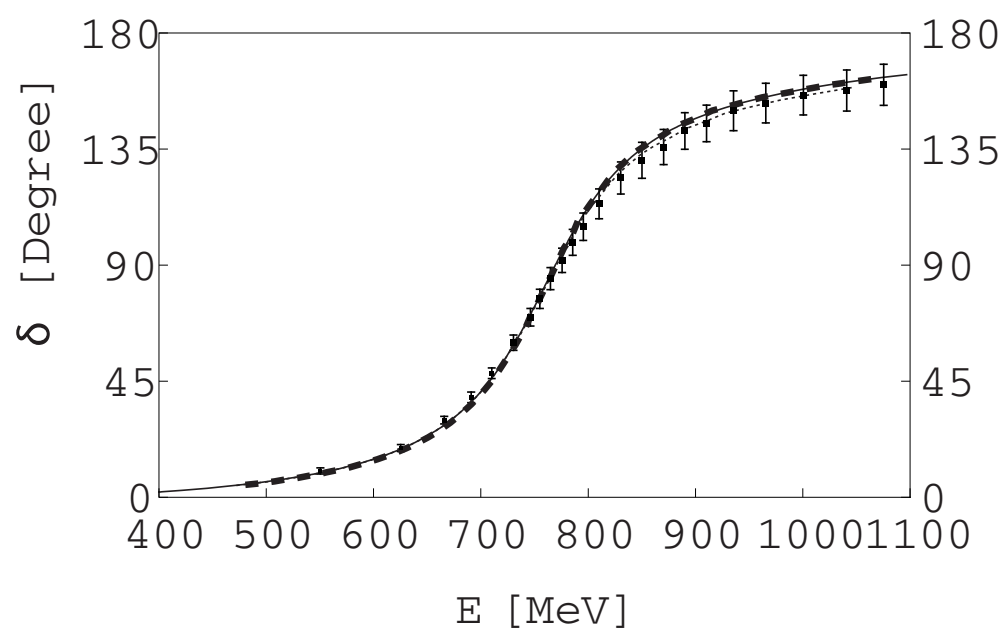

Fig. 2. The $\pi \pi$ scattering $P$-wave phase shift calculated using the chiral unitary approach, in the infinite space (solid curve) and in the finite space (dashed curve).

dashed curve is the phase shift extracted from the first energy level of the right figure of Fig. 1, obtained using only the $\pi \pi$ channel. The experimental data are taken from Ref. 41.

Phase shifts can be used to fit the physical quantities, such as $m_{\rho}, g_{\rho \pi \pi}$ and $\Gamma_{\rho}$, through the following two equations:

$$
\cot \delta(s)=\frac{m_{\rho}^{2}-s}{\sqrt{s} \Gamma_{\rho}(s)}, \quad \text { and } \quad \Gamma_{\rho}(s)=\frac{p^{3}}{s} \frac{g_{\rho \pi \pi}^{2}}{8 \pi} .
$$

We note that the factor $8 \pi$ in the second equation is our normalization, while in Refs. 21 the authors use $6 \pi$. The results from fitting the $\pi \pi$ channel are

$$
m_{\rho}=768.6 \mathrm{MeV}, g_{\rho \pi \pi}=6.59, \Gamma_{\rho}=135 \mathrm{MeV},
$$

while those from fitting the coupled channels are

$$
m_{\rho}=769.6 \mathrm{MeV}, g_{\rho \pi \pi}=6.79, \Gamma_{\rho}=144 \mathrm{MeV} .
$$

To compare our results with the lattice QCD results, ${ }^{21}$ we study the mass and decay width of the $\rho$ meson using different pion masses. We define $m_{\pi}^{0}$ to be the physical $\pi$ mass, and now $m_{\pi}$ is a free parameter. It changes from $m_{\pi}^{0}$ to $3 m_{\pi}^{0}$ in our study. When changing $m_{\pi}$, other parameters in our previous formulae also change. For the parameter $f\left(f_{\pi}\right)$ we find a good fit to results obtained in Refs. 42, 43, 44, 45, 46:

$$
\frac{f_{\pi}\left(m_{\pi}\right)}{f_{\pi}\left(m_{\pi}^{0}\right)}=1+0.035\left(\left(\frac{m_{\pi}}{m_{\pi}^{0}}\right)^{2}-1\right)
$$

with $f_{\pi}\left(m_{\pi}^{0}\right)=87.4 \mathrm{MeV}$ as we needed in our fit to the $\rho$ data. The coupling $G_{V}$ with the vector meson dominance assumption of Refs. 47,48 is related to $f_{\pi},{ }^{49}$ as 
$G_{V}=f_{\pi} / \sqrt{2}$. Therefore, we still take the value $G_{V}=53 \mathrm{MeV}$ of Refs. 37 but assume it to be proportional to $f_{\pi}$ as a function of $m_{\pi}$. On the other hand, the bare $\rho$ mass, $M_{\rho}$ in Eqs. (15), provides the link of the theory to a genuine component of the $\rho$ meson, not related to the pion cloud, and we assume it to be $m_{\pi}$ independent.

We compare our results with the Lattice results of Ref. 21:

\begin{tabular}{c|c}
\hline \hline Our Input and Result & Input and Result in Ref. 21 \\
\hline \hline$m_{\pi}=276 \mathrm{MeV}, L=2.75 m_{\pi}^{-1}=1.96 \mathrm{fm}$ & $m_{\pi}=266 \mathrm{MeV}, L=1.98 \mathrm{fm}$ \\
\hline$E_{1}=813.2_{-7.3}^{+7.2} \mathrm{MeV}$, & $E_{1}=813.4 \pm 6.3 \mathrm{MeV}$, \\
$E_{2}=1390.5_{-1.0}^{+1.0} \mathrm{MeV}$ & $E_{2}=1433.7 \pm 16.1 \mathrm{MeV}$ \\
\hline$\delta_{1}=136.3_{-1.4^{\circ}, \delta_{2}=175.6^{\circ}{ }_{-0.3^{\circ}}^{\circ}}^{\circ}$ & $\delta_{1}=130.56^{\circ} \pm 1.37^{\circ}, \delta_{2}=146.03^{\circ} \pm 6.58^{\circ}$ \\
\hline \hline
\end{tabular}

We find that the first (lowest) energy level and the extracted phase shift from the two approaches are very similar, while those from the second energy level have small differences.

\section{Energy Levels for the $\pi K$ System}

In this section we discuss the $\pi K$ system in a finite volume. The scattering amplitude has been obtained in Refs. 36, 38 using the chiral unitary model. They use the single channel Bethe-Salpeter equation:

$$
t(s)=\frac{v(s)}{1-v(s) G(s)} .
$$

The relevant $v$ matrix for $\pi K$ scattering has been studied in Ref. 38:

$$
v(s)=-\frac{1}{2 f^{2}}\left(1+\frac{2 G_{V}^{2}}{f^{2}} \frac{s}{M_{K^{*}}^{2}-s}\right) .
$$

Here the loop function $G(s)$ is given by

$$
G(s)=\int_{|\vec{q}|<q_{\max }} \frac{d^{3} \vec{q}}{(2 \pi)^{3}} \frac{|\vec{q}|^{2}}{s-\left(\omega_{1}+\omega_{2}\right)^{2}+i \epsilon} \frac{\omega_{1}+\omega_{2}}{2 \omega_{1} \omega_{2}},
$$

where $\omega_{i}=\sqrt{m_{i}^{2}+\vec{q}^{2}}$ and $i=\pi$ and $K$.

To study the $K^{*}$ meson in a finite volume, we just need to change it with the $G$ function defined in the finite box of side $L$. Energy levels for the $\pi K$ System in the finite volume are then obtained when $1-v(s) G(s)$ is zero. The detailed analyses will be shown in our future work.

\section{Conclusion}

We have made a study of the $\pi \pi$ interaction in $P$-waves in a finite volume using the chiral unitary approach. We obtained energy energies of the $\pi \pi$ system in the $\rho$ channel in a finite volume, and evaluated the relevant phase shifts. These results are compared with the Lattice results of Ref. 21, and we found that the first (lowest) 
energy level and the extracted phase shift from the two approaches are very similar, while those from the second energy level have small differences. We also discussed the $\pi K$ system in the $K^{*}$ channel in a finite volume.

\section{Acknowledgments}

This work is partly supported by DGICYT contract FIS2011-28853-C02-01, the Generalitat Valenciana in the program Prometeo, 2009/090, the EU Integrated Infrastructure Initiative Hadron Physics 3 Project under Grant Agreement no. 283286, the National Natural Science Foundation of China under Grant No. 11205011, and the Fundamental Research Funds for the Central Universities.

\section{References}

1. Y. Nakahara, M. Asakawa and T. Hatsuda, Phys. Rev. D 60, 091503 (1999).

2. N. Mathur, A. Alexandru, Y. Chen, S. J. Dong, T. Draper, I. Horvath, F. X. Lee and K. F. Liu et al., Phys. Rev. D 76, 114505 (2007).

3. S. Basak, R. G. Edwards, G. T. Fleming, K. J. Juge, A. Lichtl, C. Morningstar, D. G. Richards and I. Sato et al., Phys. Rev. D 76, 074504 (2007).

4. M. G. Alford and R. L. Jaffe, Nucl. Phys. B 578, 367 (2000).

5. A. Hart et al. [UKQCD Collaboration], Phys. Rev. D 74, 114504 (2006).

6. H. Wada, T. Kunihiro, S. Muroya, A. Nakamura, C. Nonaka and M. Sekiguchi, Phys. Lett. B 652, 250 (2007).

7. S. Prelovsek, C. Dawson, T. Izubuchi, K. Orginos and A. Soni, Phys. Rev. D 70, 094503 (2004).

8. H. -W. Lin et al. [Hadron Spectrum Collaboration], Phys. Rev. D 79, 034502 (2009).

9. C. Gattringer, C. Hagen, C. B. Lang, M. Limmer, D. Mohler and A. Schafer, Phys. Rev. D 79, 054501 (2009).

10. G. P. Engel et al. [BGR [Bern-Graz-Regensburg] Collaboration], Phys. Rev. D 82, 034505 (2010).

11. R. G. Edwards, J. J. Dudek, D. G. Richards and S. J. Wallace, Phys. Rev. D 84, 074508 (2011).

12. C. McNeile et al. [UKQCD Collaboration], Phys. Lett. B 556, 177 (2003).

13. M. Lüscher, Commun. Math. Phys. 105 (1986) 153.

14. M. Lüscher, Nucl. Phys. B 354 (1991) 531.

15. V. Bernard, M. Lage, U. -G. Meissner, A. Rusetsky, JHEP 0808, 024 (2008).

16. X. Feng et al. [ETM Collaboration], PoS LAT 2010, 104 (2010).

17. S. Aoki et al. [CP-PACS Collaboration], Phys. Rev. D 76, 094506 (2007).

18. M. Gockeler et al. [QCDSF Collaboration], PoS LATTICE 2008, 136 (2008).

19. S. Aoki et al. [PACS-CS Collaboration], PoS LATTICE 2010, 108 (2010).

20. J. Frison et al. [Budapest-Marseille-Wuppertal Collaboration], PoS LATTICE 2010, 139 (2010).

21. C. B. Lang, D. Mohler, S. Prelovsek and M. Vidmar, Phys. Rev. D 84, 054503 (2011).

22. C. B. Lang, L. Leskovec, D. Mohler and S. Prelovsek, Phys. Rev. D 86, 054508 (2012).

23. Z. Fu and K. Fu, Phys. Rev. D 86, 094507 (2012).

24. H. -X. Chen and E. Oset, Phys. Rev. D 87, 016014 (2013).

25. M. Doring, U. -G. Meissner, E. Oset and A. Rusetsky, Eur. Phys. J. A 47, 139 (2011).

26. M. Doring, J. Haidenbauer, U. -G. Meissner, A. Rusetsky, Eur. Phys. J. A 47, 163 (2011). 
27. A. Martinez Torres, L. R. Dai, C. Koren, D. Jido and E. Oset, Phys. Rev. D 85, 014027 (2012).

28. M. Doring, U. G. Meissner, JHEP 1201, 009 (2012).

29. L. Roca and E. Oset, Phys. Rev. D 85, 054507 (2012).

30. M. Albaladejo, J. A. Oller, E. Oset, G. Rios and L. Roca, JHEP 1208, 071 (2012).

31. M. Albaladejo, C. Hidalgo-Duque, J. Nieves and E. Oset, Phys. Rev. D 88, 014510 (2013).

32. M. Albaladejo, G. Rios, J. A. Oller and L. Roca, arXiv:1307.5169 [hep-lat].

33. E. J. Garzon, R. Molina, A. Hosaka and E. Oset, arXiv:1310.0972 [hep-lat].

34. J. -J. Xie and E. Oset, Eur. Phys. J. A 48, 146 (2012).

35. F. Aceti and E. Oset, Phys. Rev. D 86, 014012 (2012).

36. J. A. Oller and E. Oset, Phys. Rev. D 60, 074023 (1999).

37. J. A. Oller, E. Oset and J. E. Palomar, Phys. Rev. D 63, 114009 (2001).

38. C. W. Xiao, F. Aceti and M. Bayar, Eur. Phys. J. A 49, 22 (2013).

39. J. Gasser and H. Leutwyler, Nucl. Phys. B 250, 465 (1985).

40. G. Ecker, J. Gasser, A. Pich and E. de Rafael, Nucl. Phys. B 321, 311 (1989).

41. P. Estabrooks and A. D. Martin, Nucl. Phys. B 79, 301 (1974).

42. J. R. Pelaez and G. Rios, Phys. Rev. D 82, 114002 (2010).

43. C. Hanhart, J. R. Pelaez and G. Rios, Phys. Rev. Lett. 100, 152001 (2008).

44. P. Boucaud et al. [ETM Collaboration], Phys. Lett. B 650, 304 (2007).

45. S. R. Beane, T. C. Luu, K. Orginos, A. Parreno, M. J. Savage, A. Torok and A. WalkerLoud, Phys. Rev. D 77, 014505 (2008).

46. J. Noaki, S. Aoki, T. W. Chiu, H. Fukaya, S. Hashimoto, T. H. Hsieh, T. Kaneko and H. Matsufuru et al., PoS LATTICE 2008, 107 (2008).

47. J.J. Sakurai, Currents and mesons (University of Chicago Press, Chicago Il 1969).

48. M. Bando, T. Kugo and K. Yamawaki, Phys. Rept. 164, 217 (1988).

49. G. Ecker, J. Gasser, H. Leutwyler, A. Pich and E. de Rafael, Phys. Lett. B 223, 425 (1989). 